\title{
The computational simulation of brain connectivity using diffusion tensor MRI
Q. $\mathrm{Yu}^{1}$
F. $\mathrm{Liu}^{2}$
I. Turner ${ }^{3}$
V. Vegh ${ }^{4}$

(Received 27 January 2011; revised 14 April 2011)

\begin{abstract}
Water molecule diffusion in the brain is measured using a magnetic resonance imaging method. The anisotropy of the diffusion tensor is of particular interest in brain images, as it relates to white matter fibre tracts. Depending on the interrelation of eigenvalues, diffusion can be divided into the three cases of linear diffusion, planar diffusion and spherical diffusion. We present additional information from the (brain image) diffusion tensor magnetic resonance imaging of a patient with Parkinson's disease. This information includes maps of diffusion tensor components, fractional anisotropy and an fractional anisotropy weighted colour coded orientation. We also investigate linear diffusion, time fractional diffusion, and space fractional diffusion, as well as proposing computational simulations of connectivity in the brain using numerical methods for the analysis of diffusion tensor magnetic resonance imaging.
\end{abstract}

http://anziamj.austms.org.au/ojs/index.php/ANZIAMJ/article/view/3931 gives this article, (c) Austral. Mathematical Soc. 2011. Published April 19, 2011. ISSN 1446-8735. (Print two pages per sheet of paper.) Copies of this article must not be made otherwise available on the internet; instead link directly to this URL for this article. 


\section{Contents}

1 Introduction

C19

2 Data acquisition

C21

3 Application

C22

3.1 Diffusion tensor components . . . . . . . . . . . . C22

3.2 Anisotropies . . . . . . . . . . . . . . C C27

3.3 Fitting white matter FA frequency . . . . . . . C27

4 One-dimensional models $\quad$ C30

4.1 Model 1: Linear diffusion . . . . . . . . . . . . . . . C31

4.2 Model 2: Anomalous subdiffusion . . . . . . . . . . . C C31

4.3 Model 3: Space fractional diffusion . . . . . . . . . . C33

5 Conclusions

C35

References

C35

\section{Introduction}

Diffusion tensor magnetic resonance imaging (DT-MRI) is a technique used to measure the diffusion properties of water molecules in tissues [1]. In white matter, which is one of the two components of the central nervous system and consists mostly of myelinated axons, the diffusion of free water molecules is anisotropic and such diffusion is often modelled by the equation

$$
\frac{\partial \mathrm{C}}{\partial \mathrm{t}}=\nabla \cdot(\mathrm{D} \nabla \mathrm{C})
$$

where $C(x, y, z, t)$ is the concentration of water molecules and $D(x, y, z)$ is the usual symmetric second rank diffusion tensor. 
A property of symmetric second rank tensors is that they can always be orthogonally diagonalised as $\mathrm{D}=\mathrm{E} \wedge \mathrm{E}^{\top}=\sum_{i=1}^{3} \lambda_{i} e_{i} e_{i}^{\top}$ with $\mathrm{E}=\left[e_{1}, e_{2}, e_{3}\right]$ and $\Lambda=\operatorname{diag}\left(\lambda_{1}, \lambda_{2}, \lambda_{3}\right)[2]$. The eigenvalues reflect the shape or configuration of an ellipsoid with its surface representing the root mean square diffusive displacement of free water in anisotropic media. The tensor trace equals the sum of the eigenvalues, $\lambda_{1}+\lambda_{2}+\lambda_{3}$, and is rotationally invariant, that is, independent of the orientation of the ellipsoid. The tensor trace reflects the size of the ellipsoid, whereas the eigenvectors $e_{1}, e_{2}$ and $e_{3}$ describe the relationship between the ellipsoid and the laboratory frame.

In general, the eigenvalues are sorted with the convention that $\lambda_{1} \geqslant \lambda_{2} \geqslant \lambda_{3}$. Consequently, the first eigenvector $e_{1}$ describes the predominant diffusion direction and is therefore also called the principal diffusion vector or principal diffusivity.

Depending on the interrelation of the eigenvalues $\lambda_{i}$ of $D$ at each locale, diffusion is divided into three cases [3]:

1. linear diffusion $\lambda_{1} \gg \lambda_{2} \approx \lambda_{3}$ and $\mathrm{D} \approx \lambda_{1} \mathrm{e}_{1} \mathrm{e}_{1}^{\mathrm{T}}$;

2. planar diffusion $\lambda_{1} \approx \lambda_{2} \gg \lambda_{3}$ and $\mathrm{D} \approx \lambda_{1} \sum_{i=1}^{2} e_{i} e_{i}^{\top}$;

3. spherical diffusion $\lambda_{1} \approx \lambda_{2} \approx \lambda_{3}$ and $\mathrm{D} \approx \lambda_{1} \sum_{i=1}^{3} e_{i} e_{i}^{\top}$.

The anisotropy captured using diffusion tensor imaging is of particular interest in relation to the brain, as it is associated with white matter fibre tracts. Several measures of diffusion anisotropy, including fractional anisotropy (FA), relative anisotropy, and volume ratio, are calculated from the eigenvalues. Throughout this article we use [4]

$$
\mathrm{FA}=\frac{1}{\sqrt{2}} \frac{\sqrt{\left(\lambda_{1}-\lambda_{2}\right)^{2}+\left(\lambda_{2}-\lambda_{3}\right)^{2}+\left(\lambda_{3}-\lambda_{1}\right)^{2}}}{\sqrt{\lambda_{1}^{2}+\lambda_{2}^{2}+\lambda_{3}^{2}}} .
$$

Figure 5 exhibits the orientation map images showing the direction of the maximum eigenvector. The colours given in this figure are chosen according to the scheme recommended by Sarntinoranont et al. [5]. The maps are 
coloured according to the eigenvector direction and scaled by multiplying the eigenvector direction cosine with the fractional anisotropy.

Several recent studies investigated so called anomalous diffusion $[6,7,8]$. We consider an alternative derivation for the stretched diffusion model using fractional order space and time derivatives.

We first exhibit information derived from the diffusion tensor, which includes the maps of diffusion tensor components, the eigenvalues and the FA weighted color coded orientation. Then models relating to linear diffusion are presented. Numerical simulations of the linear diffusion, time fractional diffusion and space fractional diffusion are investigated.

\section{Data acquisition}

The pre and post surgery data we use here is from a patient diagnosed with Parkinson's disease. The brain diffusion tensor MR images are acquired using a GE Medical System (SIGNA 3T) scanner. All image pixels are $256 \times 256$.

Pre surgery data is acquired using an echo time of $93.7 \mathrm{~ms}$ and repetition time of $7 \mathrm{~s}$. Twenty four interleaved, $5 \mathrm{~mm}$ thick slices are acquired in the horizontal plane perpendicular to the coronal and sagittal planes, using the multislice mode. Diffusion sensitization is performed along 35 different diffusion gradient orientations using a diffusion weighting of $b=1000 \mathrm{~s} / \mathrm{mm}^{2}$. A reference image without diffusion weighting $\left(b=0 \mathrm{~s} / \mathrm{mm}^{2}\right)$ was also recorded.

The patient underwent deep brain stimulation. Post surgery data is acquired using an echo time of $93.5 \mathrm{~ms}$ and repetition time of $7 \mathrm{~s}$. Twenty six interleaved, $5 \mathrm{~mm}$ thick slices are acquired in the horizontal plane using the multislice mode. Furthermore, diffusion sensitization is performed along 40 different diffusion gradient orientations using a diffusion weighting of $\mathrm{b}=1000 \mathrm{~s} / \mathrm{mm}^{2}$. Similary, a reference image without diffusion weighting $\left(\mathrm{b}=0 \mathrm{~s} / \mathrm{mm}^{2}\right)$ was also recorded. 


\section{Application}

\subsection{Diffusion tensor components}

The images shown in Figures 1 and 2 depict the diffusion tensor components $\left(D_{x x}, D_{x y}, D_{x z}, D_{y y}, D_{y z}\right.$ and $\left.D_{z z}\right)$ determined pre and post surgery, respectively. Note the changes in the tensor components after surgery are no longer evident, particularly in the diagonal entries where the distinct central structure.

Figure 3 depicts the changes in the eigenvalues after surgery. The largest, middle and smallest eigenvalues are shown before surgery in (a), (b) and (c) and after surgery in (d), (e) and (f) respectively. The intervention had a significant impact on the eigenvalue distributions, again most notably around the central structure. Further details on classifying changes in the brain images via the magnitudes of the eigenvalues was discussed by Leemans [3].

Figure 4(a) and (b) display images of the brain before and after surgery respectively. Table 1 presents the relevant data. In addition, in Figure 4(a) and (b) we mark locations $\mathrm{A}, \mathrm{B}$ and $\mathrm{C}$ based on the diffusion cases identified according to the magnitude of the eigenvalues. Point A, before surgery, could be classified as linear diffusion, because the largest eigenvalue is approximately five times that of the other eigenvalues, whereas after surgery all eigenvalues have approximately the same magnitude indicating a spherical diffusion classification. Also, point B changes from spherical to planar diffusion after surgery, whereas point $\mathrm{C}$ is unchanged. These findings may be helpful to the surgeon treating the patient, and further analysis of this data will be carried out in conjunction with a medical specialist in our future work. 

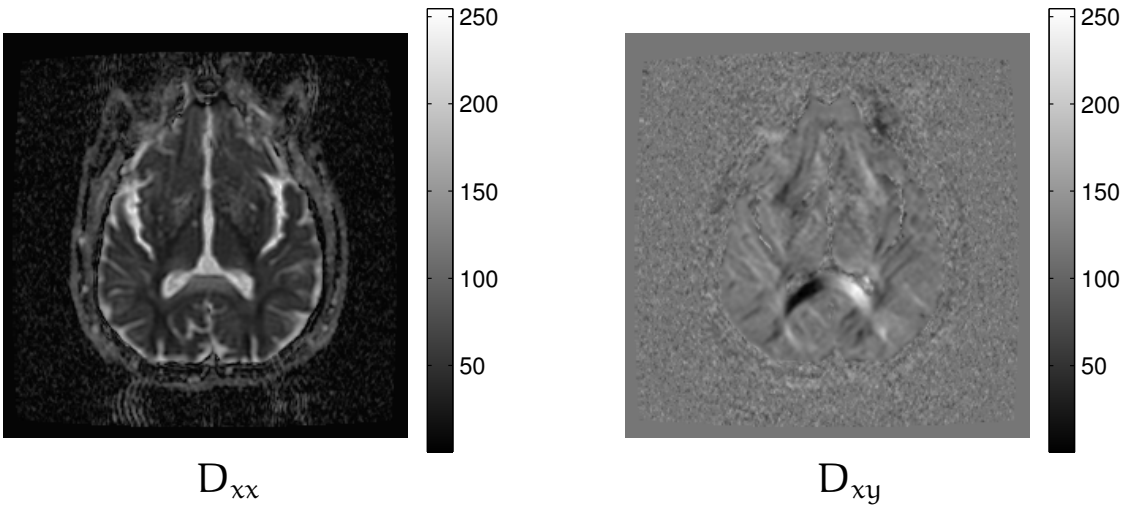

250

200

150

100

50

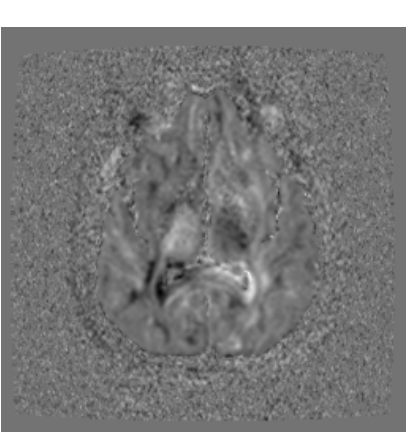

$-250$

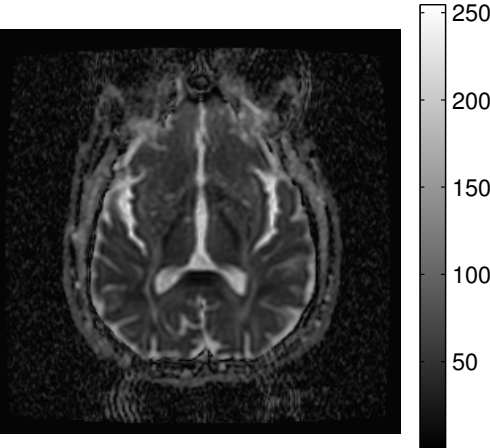

$\mathrm{D}_{x z}$

$\mathrm{D}_{y y}$
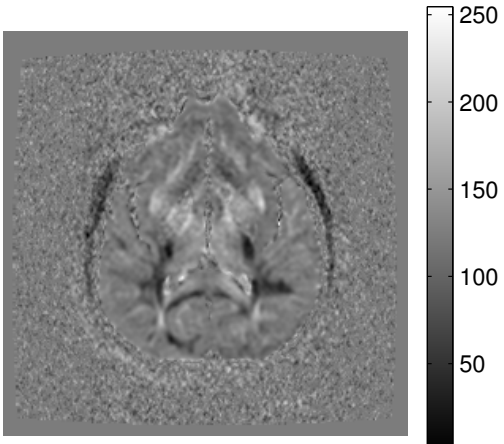

$-200$

$\mathrm{D}_{y z}$

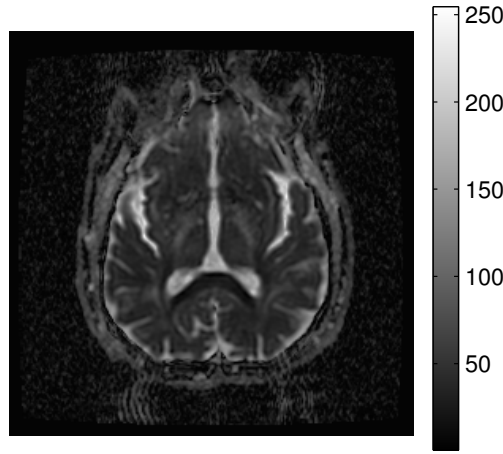

$\mathrm{D}_{z z}$

FiguRE 1: Diffusion tensor components before surgery. 

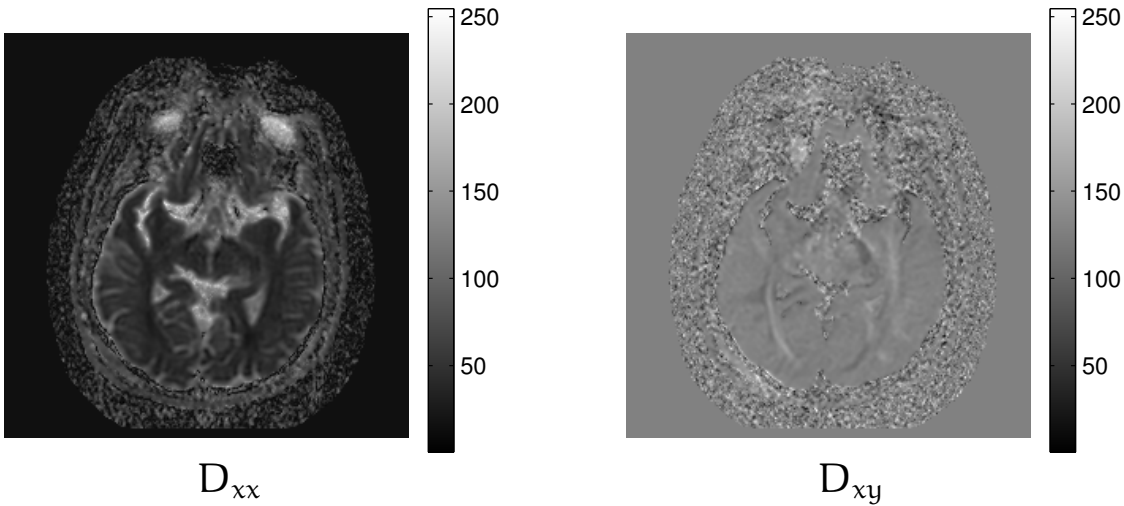

250

200

150

100

50
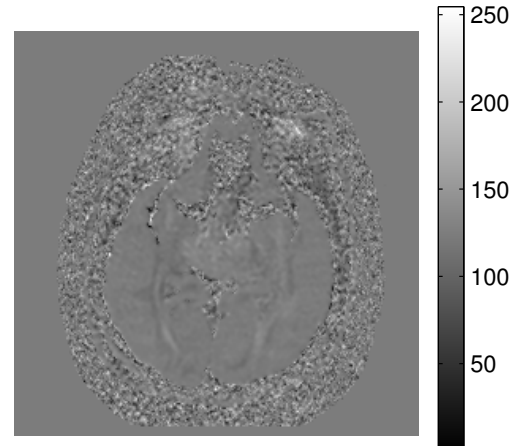

$-200$

150

100

50

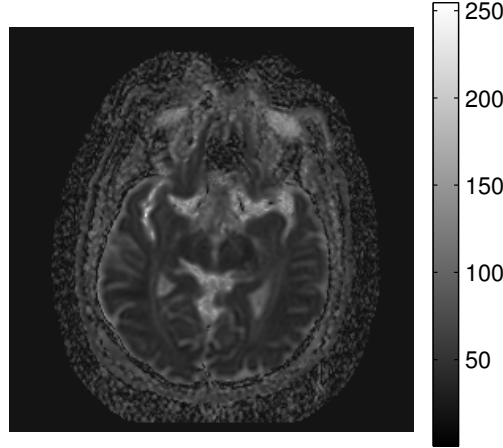

$\mathrm{D}_{y y}$
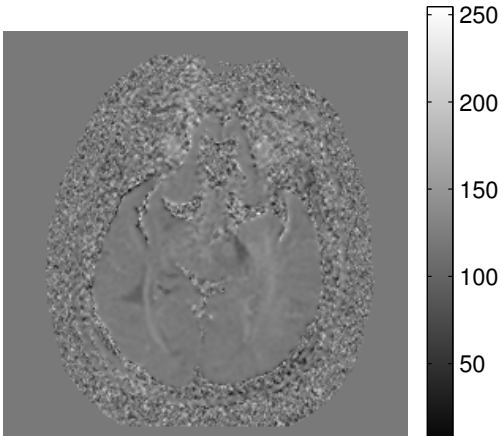

$-7250$

200

150

100

50

$\mathrm{D}_{\mathrm{yz}}$

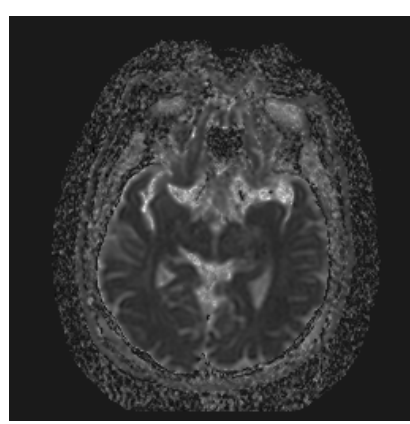

250

$-200$

$-150$

$-100$

$\mathrm{D}_{z z}$

Figure 2: Diffusion tensor components after surgery. 

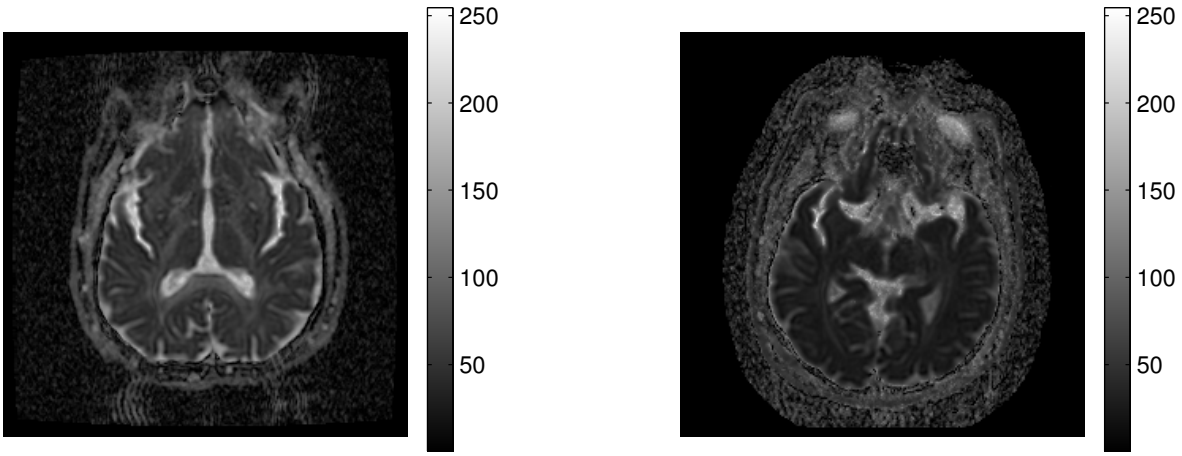

(a)

(d)
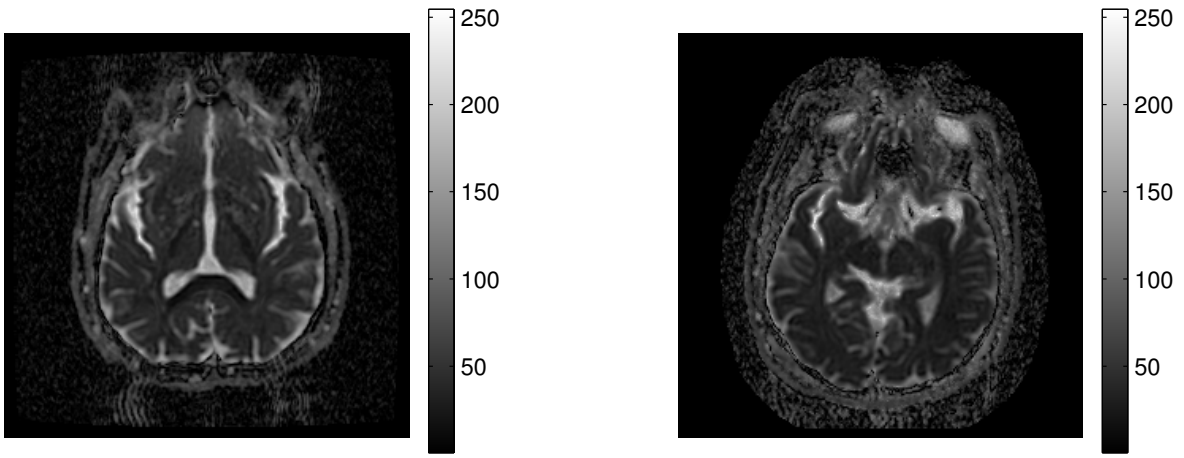

(b)

(e)
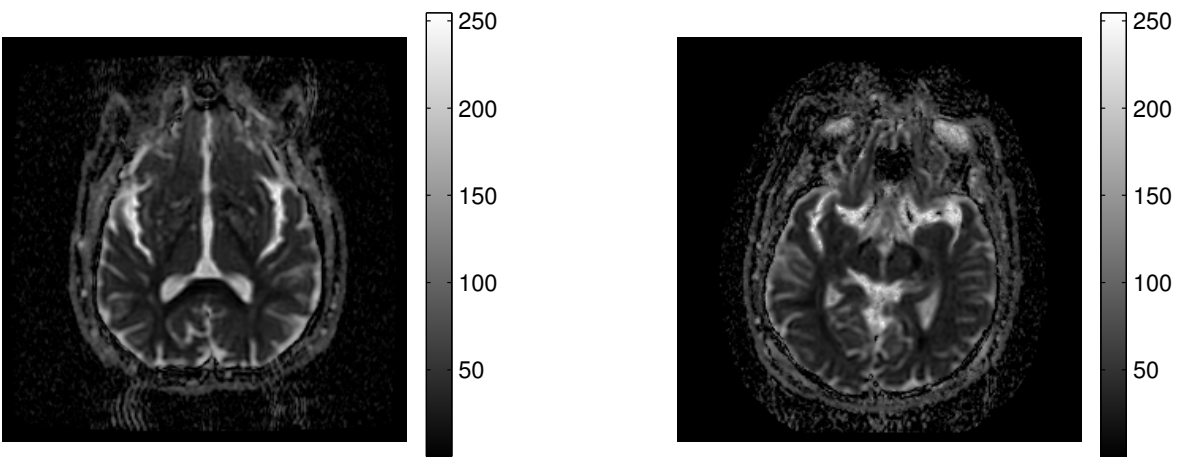

(c)

(f)

Figure 3: Eigenvalues: left column is pre-surgical; and right column is post-surgical. 

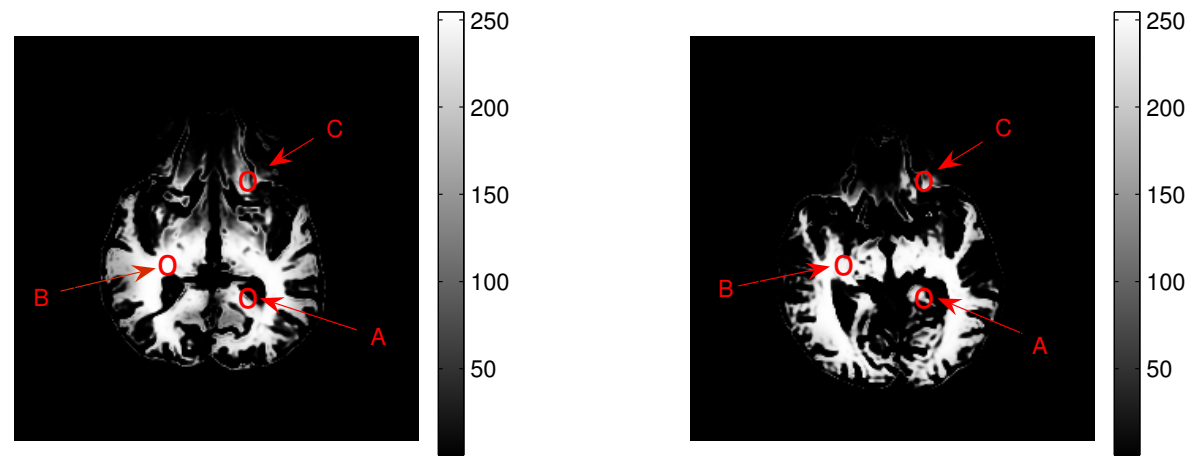

(a)

(b)

Figure 4: Three eigenvalues in white matter, (a) pre surgical and (b) post surgical.

TABLE 1: Eigenvalues at the same points in white matter (WM) before and after surgery. All eigenvalues need to be multiplied by $10^{-3}$.

\begin{tabular}{lcc}
\hline WM & Eigenvalues(pre surgical) & Eigenvalues(post surgical) \\
\hline $\mathrm{A}(142,164)$ & $(1.661,0.308,0.251)$ & $(1.337,1.030,0.922)$ \\
$\mathrm{B}(91,143)$ & $(0.606,0.559,0.422)$ & $(1.100,1.050,0.550)$ \\
$\mathrm{C}(142,90)$ & $(1.169,0.841,0.520)$ & $(2.392,1.276,0.633)$ \\
\hline
\end{tabular}




\subsection{Anisotropies}

Figure 5 shows color coded orientation map images representing the direction of the maximum eigenvector with its intensity weighted by FA. The color coded orientation maps with the largest, middle and smallest eigenvalues are shown before surgery in (a), (b) and (c) and after surgery in (d), (e) and (f) respectively. In this figure, red represents the eigenvector direction perpendicular to the image plane, green represents left to right, and blue represents up and down. The surgical procedure has altered the maximum eigenvector direction distributions.

Figure 6(c) shows the mean FA values of the patient were lower before surgery. However, the mean FA values of a normal adult are $0.68 \pm 0.18$. The data of a normal adult are reproduced from the book by Mori [4]. Figure 6(c) shows that the surgery produces a mean FA value closer to that observed for a normal adult, but this conclusion would be subject to analysis by a medical specialist.

\subsection{Fitting white matter FA frequency}

Using the results of Zhao et al. [9], we found that a mixture of two distributions, a normal distribution and the inverse Gaussian distribution, given by the formula (5), can be used to fit the distribution of the whole brain white matter fractional anisotropy (FA) frequency. Letting the probability density functions $f_{1}(x)$ and $f_{2}(x)$ be defined as

$$
\begin{aligned}
& f_{1}(x)=\frac{1}{\sqrt{2 \pi \sigma^{2}}} \exp \left[\frac{-\left(x-\mu_{1}\right)^{2}}{2 \sigma^{2}}\right], \quad x \in[a, b], \\
& f_{2}(x)=\sqrt{\frac{\lambda}{2 \pi x^{3}}} \exp \left[\frac{-\lambda\left(x-\mu_{2}\right)^{2}}{2 \mu^{2}} x\right], \quad x \in[c, d],
\end{aligned}
$$




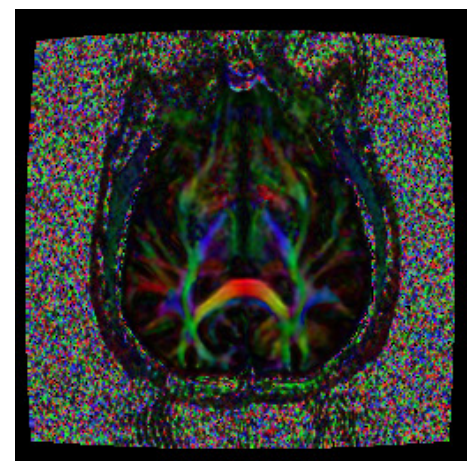

(a)

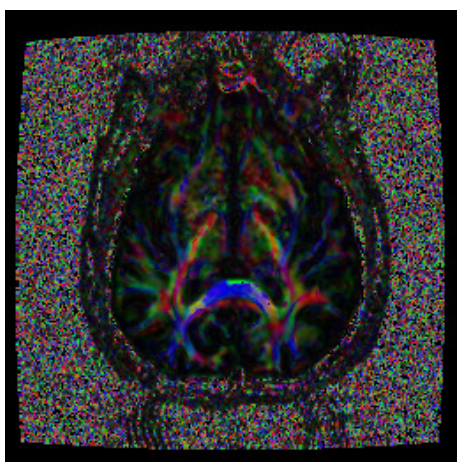

(b)

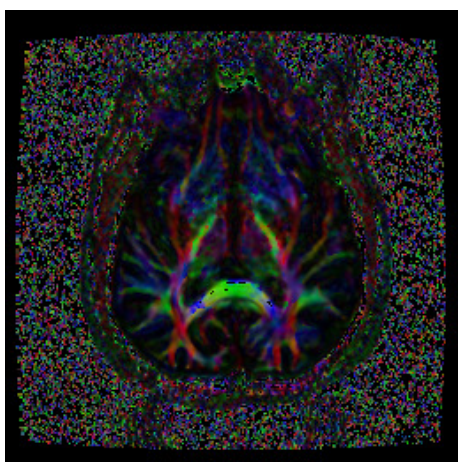

(c)

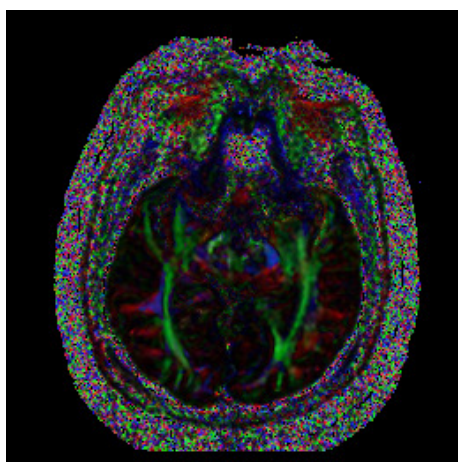

(d)

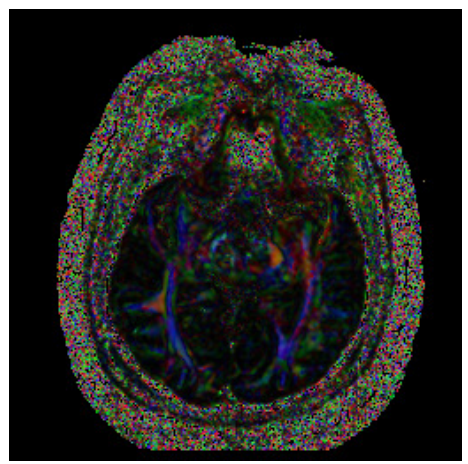

(e)

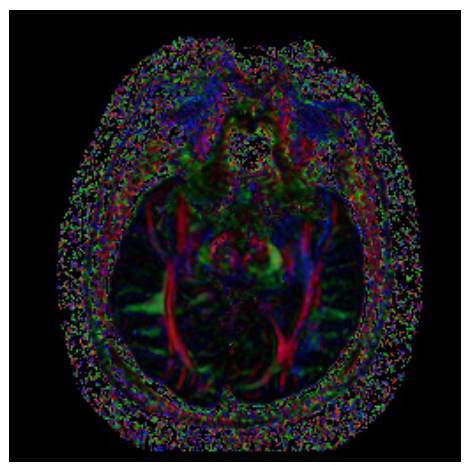

(f)

FiguRE 5: FA weighted color coded orientation maps: left column is pre surgical; and right column is post surgical. 
then the function $f(x)$ is defined as

$$
f(x)= \begin{cases}f_{1}(x) / 2, & x \in[a, b], x \notin[c, d] \\ \left(f_{1}(x)+f_{2}(x)\right) / 2, & x \in[a, b] \cap[c, d] \\ f_{2}(x) / 2, & x \in[c, d], x \notin[a, b]\end{cases}
$$

Figures $6(\mathrm{a})([\mathrm{a}, \mathrm{b}]=[0,0.7]$ and $[\mathrm{c}, \mathrm{d}]=[0.05,1])$ and $(\mathrm{b})([\mathrm{a}, \mathrm{b}]=[0,0.35]$ and $[c, d]=[0.1,1])$ show the fitted results for the pre surgical and post surgical whole brain white matter fractional anisotropy frequency respectively. We evaluate the accuracy of the fitted results based on the relative standard error $e=e_{1} / e_{2}$, where

$$
e_{1}=\sqrt{\frac{1}{N} \sum_{i=1}^{N}\left(f_{d}\left(x_{i}\right)-\hat{f}\left(x_{i}\right)\right)^{2}}, \quad e_{2}=\sqrt{\frac{1}{N} \sum_{i=1}^{N}\left(f_{d}\left(x_{i}\right)-f_{a v e}\right)^{2}} .
$$

Here $f_{d}\left(x_{i}\right)$ is the empirical PDF, with average value $f_{\text {ave }}$, and $\hat{f}(x)$ is the fitted probability density function. The goodness of fit is indicated by the result $e<1.0$ as pointed out by Zhao et al. [9]. All the values of $e$ in Figures 6(a) and (b) are smaller than 0.45 , indicating that a mixture of two distributions, defined by formula (5), performs well in fitting the distribution of whole brain white matter fractional anisotropy frequency.

Figures 6(a) and (b) show the post surgical whole brain white matter fractional anisotropy distribution (post WM-FAD) to be positively skewed and leptokurtic, whereas the pre surgical whole brain white matter fractional anisotropy distribution (pre WM-FAD) is positively skewed and less leptokurtic than the post WM-FAD. Furthermore, there is an interaction between the measured histogram parameters, see for example the post WM-FAD, which is highly leptokurtic having a greater peak height frequency than the pre WM-FAD, while the post WM-FAD also has a greater positive skew being more asymmetrical than the pre WM-FAD. 


\section{One-dimensional models}

When the diffusion is linear, planar and spherical, the corresponding diffusion equation (1) becomes one dimensional, two dimensional and three dimensional respectively.

The distribution and dynamics of water in biological tissues is wholly unobservable to conventional NMR and DT-MRI systems [10]. This invisibility is not solely due to signal to noise ratio (SNR) or image resolution issues, but is fundamentally encoded in the definition of magnetisation as a spatial average of the magnetic moment per unit volume, and the central limit theorem model of a Gaussian space/time averaging of the phase of the detected NMR signal (free induction decay) - typically the phase of the transverse components of the bulk magnetisation. The main characteristic of a fractional model is that it contains a non-integer order derivative. Fractional models can effectively describe memory and transmissibility of many kinds of material, and play an increasingly important role in engineering, physics, finance, hydrology and other fields. Fractional calculus may play a similar role in the analysis of MR images of complex biological tissues. Several authors demonstrated that a fractional calculus based diffusion model successfully applies to analysing diffusion images of human brain tissue and provides new insights into further investigations of tissue structures and the microenvironment [7, 10].

This section aims to illustrate how the diffusion tensor information is used to model the diffusion behaviour in the white matter of the brain. The models we investigate are standard diffusion, anomalous subdiffusion (time fractional diffusion), and space fractional diffusion.

Recall from Section 3.1 that the spectrum of the diffusion tensor located at point A before surgery could be classified as 'linear' diffusion, and this motivates a one dimensional modelling study, which we consider throughout the following paragraphs, emanating from point A. We only show numerical results for the one dimensional model with the initial condition given by the Dirac delta function $\delta(x)$ and Neumann boundary conditions obtained 
from finite difference methods $[6,8]$. Here, a diffusion equation based on the measured diffusion tensor is solved with the initial condition taken at a location of the tractography starting point (for example, point A in Figure 4 before surgery) [1]. The two dimensional and three dimensional models can be similary simulated and their solutions will be exhibited in future work.

\subsection{Model 1: Linear diffusion}

When the diffusion is linear, that is, when $\lambda_{1} \gg \lambda_{2} \approx \lambda_{3}$, equation (1) becomes the well known standard diffusion equation (SDE):

$$
\frac{\partial \mathrm{C}}{\partial \mathrm{t}}=\mathrm{D}_{1} \frac{\partial^{2} \mathrm{C}}{\partial \xi^{2}}, \quad-\mathrm{L} \leqslant \xi \leqslant \mathrm{L}, \quad 0 \leqslant \mathrm{t} \leqslant \mathrm{T},
$$

where $t$ is the time variable, $\xi$ is the space variable, $D_{1}, L$ and $T$ are constants. This is an illustrative example that exhibits linear diffusion from say point $\mathrm{A}$ in Figure 4 before surgery where a surgical treatment is injected and the direction $\xi$ could be interpreted along the dominant eigenvector. Using the numerical method proposed by Zhuang et al. [8], the behaviour of the SDE with $L=5$ and $D_{1}=1$ is shown in Figure 6(d).

\subsection{Model 2: Anomalous subdiffusion}

Fractional kinetic equations proved particularly useful in the context of anomalous subdiffusion [11]. The mean square displacement of particles released from the original starting site is no longer linear in time and follows a generalised Fick's second law. Subdiffusive motion is characterised by an asymptotic long time behaviour of the mean square displacement of the form $\left\langle\xi^{2}(\mathrm{t})\right\rangle \sim 2 \mathrm{~K}_{\gamma} \mathrm{t}^{\gamma} / \Gamma(1+\gamma)(\mathrm{t} \rightarrow \infty)$, where $\gamma(0<\gamma<1)$ is the anomalous diffusion exponent and $\boldsymbol{K}_{\gamma}$ is the generalised diffusion coefficient.

For anomalous subdiffusive random walkers, the continuum description via the ordinary diffusion equation is replaced by the fractional diffusion equation. It 


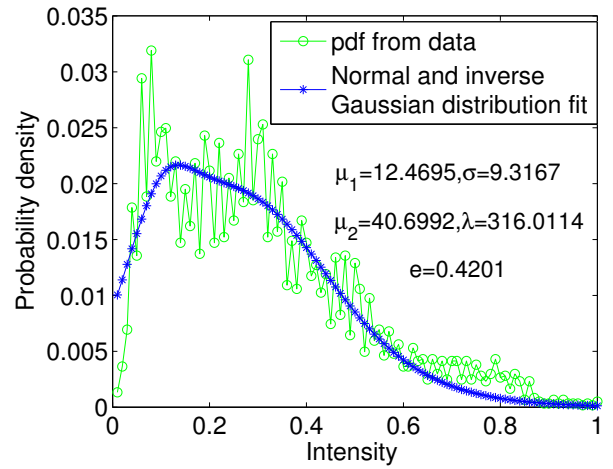

(a)

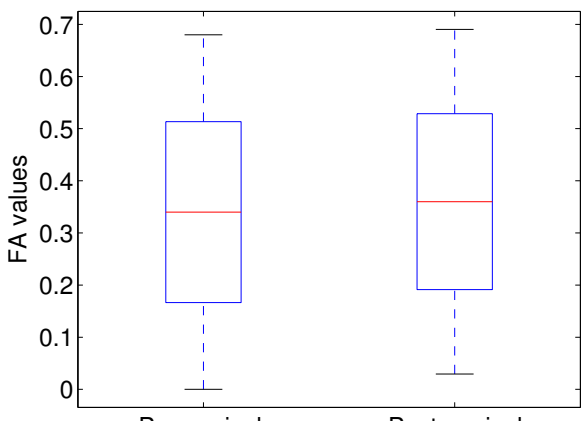

Presurgical

(c)

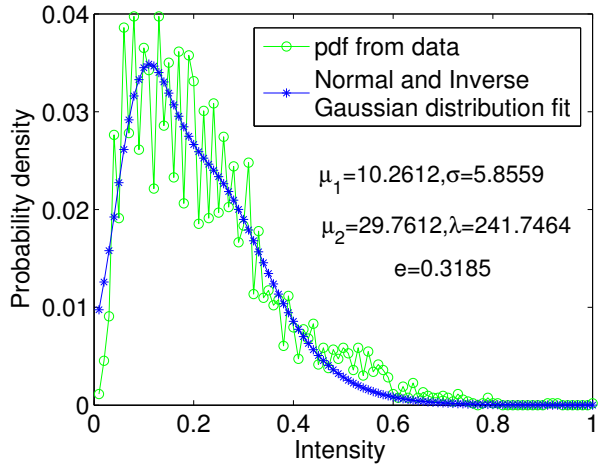

(b)

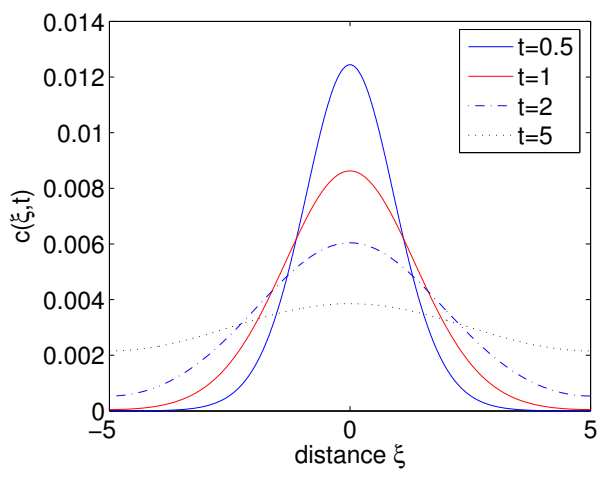

(d)

Figure 6: (a) Pre WM-FAD after fitting; (b) post WM-FAD after fitting; (c) mean FA values; (d) solution profiles of SDE as a function of $\xi$ for different $t$. 
has been suggested that the probability density function $C(\xi, t)$ that describes anomalous subdiffusion particles follows the anomalous subdiffusion equation $(\mathrm{AS}-\mathrm{DE})[11]$

$$
\frac{\partial \mathrm{C}}{\partial \mathrm{t}}=\mathrm{D}_{\mathrm{t}}^{1-\gamma}\left(\mathrm{K}_{\gamma} \frac{\partial^{2} \mathrm{C}}{\partial \xi^{2}}\right), \quad-\mathrm{L} \leqslant \xi \leqslant \mathrm{L}, \quad 0 \leqslant \mathrm{t} \leqslant \mathrm{T}, \quad 0<\gamma \leqslant 1,
$$

where $C(\xi, t)$ is the probability density and $D_{t}^{1-\gamma}$ is the Riemann-Liouville fractional derivative with respect to $t$, which is defined as [8]

$$
D_{t}^{1-\gamma} C(\xi, t)=\frac{1}{\Gamma(\gamma)} \frac{\partial}{\partial t} \int_{0}^{t} \frac{C(\xi, \eta) d \eta}{(t-\eta)^{1-\gamma}}, \quad 0<\gamma \leqslant 1
$$

If $\gamma \rightarrow 0$, then $\mathrm{D}_{\mathrm{t}}^{1-\gamma} \mathrm{C} \rightarrow \partial \mathrm{C} / \partial \mathrm{t}$.

Applying the numerical method given by Zhuang et al. [8], the evolution results of AS-DE with $\mathrm{L}=5, \mathrm{~K}_{\gamma}=1$ are shown in Figure 7(a). Figure 7(a) shows that the system exhibits subdiffusion behaviour, that is, the particles diffuse more slowly as $\gamma$ decreases, as to be expected, and the solution depends continuously on the time fractional derivative.

\subsection{Model 3: Space fractional diffusion}

Because white matter is highly heterogeneous, the laws of Markov diffusion [12] may be altered in a fundamental way, and so the classical diffusion equation may not be adequate. If the complex structure, such as the spatial connectivity, can facilitate movement of particles within a certain scale, fast motions may no longer obey the classical Fick's law and may have a probability density function that follows a power law. Superdiffusion is one possible form for fast motions.

Fractional derivatives play a key role in modelling particle transport in anomalous diffusion. By replacing the second space derivative by a fractional 


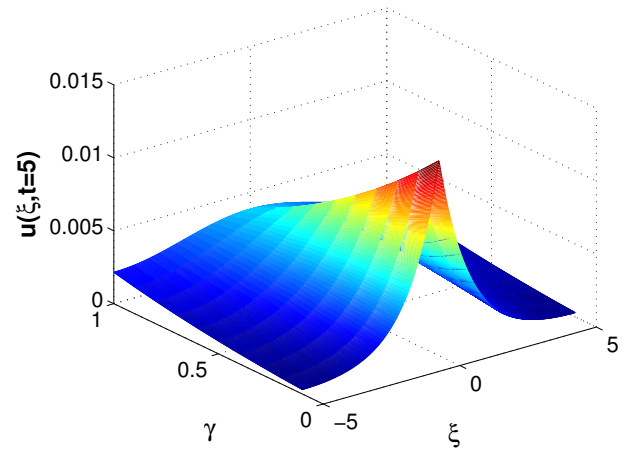

(a)

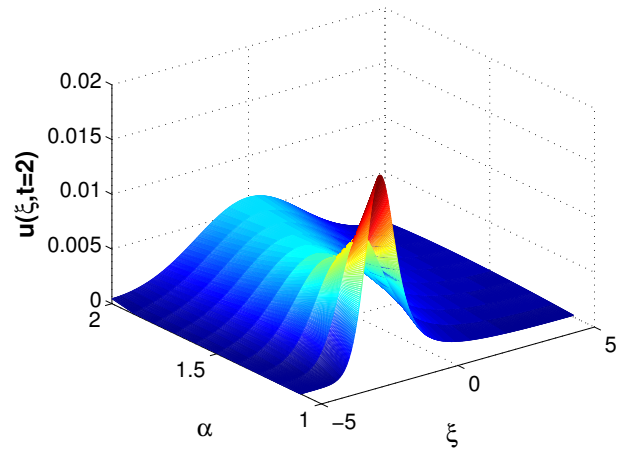

(b)

FIgURE 7: (a) Numerical results of AS-DE at $t=5$; (b) numerical results of SF-DE at $t=2$.

derivative of order $\alpha(1<\alpha \leqslant 2)$, the classical diffusion equation SDE becomes a one dimensional space fractional diffusion equation (SF-DE):

$$
\frac{\partial \mathrm{C}}{\partial \mathrm{t}}=\mathrm{D}_{2} \frac{\partial^{\alpha} \mathrm{C}}{\partial \xi^{\alpha}}, \quad-\mathrm{L} \leqslant \xi \leqslant \mathrm{L}, \quad 0 \leqslant \mathrm{t} \leqslant \mathrm{T}, \quad 1<\alpha \leqslant 2,
$$

where $\mathrm{D}_{2}$ is constant, and $\partial^{\alpha} / \partial \xi^{\alpha}$ is the Riemann-Liouville fractional derivative with respect to $\xi$, which is defined as [6]

$$
\frac{\partial^{\alpha} C(\xi, t)}{\partial \xi^{\alpha}}=\frac{1}{\Gamma(2-\alpha)} \frac{\partial^{2}}{\partial \xi^{2}} \int_{0}^{\xi} \frac{C(\eta, t) d \eta}{(\xi-\eta)^{\alpha-1}}, \quad 1 \leqslant \alpha<2 .
$$

If $\alpha \rightarrow 2$, then $\partial^{\alpha} \mathrm{C} / \partial \xi^{\alpha} \rightarrow \partial^{2} \mathrm{C} / \partial \xi^{2}$.

Using the numerical method in Liu et al. [6], Figure 7(b) shows the solution profiles of SF-DE with $L=5, D_{2}=1$. Figure $7(b)$ shows that the solution depends continuously on the order $\alpha$ of the fractional derivative in space. 


\section{Conclusions}

The diffusion tensor information from water molecules diffusing in the brain, obtained using DT-MRI, was described and demonstrated. We derived numerical methods to analyse brain images, and developed one dimensional linear diffusion and fractional models to study standard diffusion and anomalous diffusion behaviour in the white matter of the brain. In addition, brain image data was compared for a patient with Parkinson's disease before and after surgery. The simulated information can provide the surgeon with a more fundamental understanding of the impact of surgery on the diffusion behaviour in the white matter of the brain. Future research will be directed towards refining and calibrating the models presented here by using expert opinion and feedback from medical practitioners working in this field.

Acknowledgements We gratefully acknowledge the help and interest in our work by Professor Kerrie Mengersen from QUT. Mr Yu also acknowledges the Centre for Complex Dynamic Systems Control for offering financial support for his $\mathrm{PhD}$ scholarship. We also thank the referees for their constructive comments and suggestions.

\section{References}

[1] P. G. Batchelor, D. L. G. Hill, F. Calamante and D. Atkinson, Study of connectivity in the brain using the full diffusion tensor from DT-MRI. Proceedings of Information Processing in Medical Imaging IPMI, 2082: 121-133, 2001. C19, C31

[2] E. R. Melhem, S. Mori, G. Mukundan, M. A. Kraut, M. G. Pomper and P. C. M. van Zijl, Diffusion Tensor MR Imaging of the Brain and White Matter Tractography, American Journal of Roentgenology, 178: 3-16, 2002. http://www. ajronline.org/cgi/content/full/178/1/3. C20 
[3] A. Leemans, Modeling and processing of diffusion tensor magnetic resonance images for improved analysis of brain connectivity. $P h D$ thesis, University of Antwerp, Belgium, 2006. C20, C22

[4] S. Mori, Introduction to Diffusion Tensor Imaging. Elsevier B.V., 2007. C20, C27

[5] M. Sarntinoranont, X. Chen, J. Zhao and T. H. Mareci, Computational model of interstitial transport in the spinal cord using diffusion tensor imaging. Annals of Biomedical Engineering, 34(8): 1304-1321, 2006. C20

[6] F. Liu, V. Anh, I. Turner Numerical solution of the space fractional Fokker-Planck equation. Journal of Computational and Applied Mathematics, 166: 209-219, 2004. doi:10.1016/j.cam.2003.09.028 C21, C31, C34

[7] R. Magin, X. Feng, D. Baleanu, Solving the fractional order Bloch equation, Concepts in Magnetic Resonance Part A, 34A(1): 16-23, 2009. $\mathrm{C} 21, \mathrm{C} 30$

[8] P. Zhuang, F. Liu, V. Anh and I. Turner, New solution and analytical techniques of the implicit numerical methods for the anomalous sub-diffusion equation. SIAM J. on Numerical Analysis, 46(2): 1079-1095, 2008. doi:10.1137/060673114 C21, C31, C33

[9] Z. Zhao, Z. G. Yu, V. V. Anh, Topological properties and fractal dimension of the Sierpinske and generalized Sierpinski networks. Commun. Frac. Calc., 1: 15-26, 2010. C27, C29

[10] M. P. Velasco, J. Trujillo, D. A. Reiter, R. G. Spencer, W. Li, and R. L. Magin, Anomalous Fractional Order Models of NMR Relaxation. Proceedings of Fractional Differentiation and its Applications FDA'10, FDA10-058, 2010. C30 
[11] R. Metzler and J. Klafter, The random walks guide to anomalous diffusion: A fractional dynamics approach, Phys. Rep., 339: 1-77, 2000. doi:10.1016/S0370-1573(00)00070-3 C31, C33

[12] M. Ledoux, The Geometry of Markov Diffusion Generators, Annales de la Faculte des Sciences de Toulouse, 6(9): 305-366, 2000. C33

\section{Author addresses}

1. Q. Yu, Discipline of Mathematical Sciences, Queensland University of Technology, Brisbane 4001, Australia.

2. F. Liu, Discipline of Mathematical Sciences, Queensland University of Technology, Brisbane 4001, Australia. mailto:f.liu@qut.edu.au

3. I. Turner, Discipline of Mathematical Sciences, Queensland University of Technology, Brisbane 4001, Australia.

4. V. Vegh, Centre for Advanced Imaging, University of Queensland, Brisbane 4072, Australia. 